\title{
A tactile P300-based BCI for communication and detection of awareness
}

\author{
Ortner R. ${ }^{1}$, Prückl R. ${ }^{1}$, Guger C ${ }^{1}$ \\ ${ }^{1}$ g.tec Guger Technologies OG, Graz, Austria \\ ortner@gtec.at
}

\begin{abstract}
In this publication a tactile P300-based BrainComputer Interface (BCI) is presented. It can be used for communication, but is also aimed for testing the consciousness in nonresponsive patients. Three different settings were evaluated: two stimulators for testing if a P300 could be detected; three stimulators that could be used for simple communication, and a setup with eight stimulators that provides more classes and is hence suitable for advanced communication. The BCI was evaluated on 12 healthy users showing a mean accuracy of 100\% for classification in the two stimulator approach, $80 \%$ in the three stimulator approach and $69.4 \%$ in the eight stimulator approach.
\end{abstract}

Keywords: EEG, P300, vibrotactile stimulation, minimal conscious state, persistent vegetative state.

\section{Introduction}

Brain-Computer Interfaces (BCIs) for communication are usually controlled via a P300 paradigm. During the last 25 years, several P300 spellers based on visual stimulation have been developed. But for users suffering visual impairments or even in a minimally conscious state, visual stimulation cannot be used any more. For these groups of users, a tactile stimulation can be used to elicit the event related potential (ERP). Hence, one can use this way of stimulation for communication and also for assessment of the level of consciousness in patients classified as non-responsive. Kotchoubey et al. tested ERPs to stimuli of different complexity levels in patients with persistent vegetative state (PVS) and minimal conscious state [1]. They found a P300 also in PVS patients although it was associated more frequent to patients with lower level of disability.

Therefore, testing only for the existence of a P300 response is not sufficient to decide about the consciousness of the patient, but it is the first step that could lead to further tests with the proposed BCI.

Next, one should present certain commands to the patient, like selecting a specific sequence of symbols. If the user is able to follow the sequence, the patient could be classified as responsive.

For a visual P300 speller, it was shown that healthy users reach an average control accuracy of $91 \%$ [2] and a group of fifteen people suffering motor impairments reached $70.1 \%$ [3]. In this paper, we evaluate the control accuracy of a tactile P300 speller on twelve healthy users.

\section{Methods}

Three different scenarios were tested. In the first paradigm, two vibrotactile stimulators (g.VIBROstim, g.tec medical engineering, Austria, see Fig. 1) were placed on the user's wrists. One stimulator delivered a train of standard stimuli (one stimulus was a short vibration of the stimulator). The stimulator on the other wrist produced the deviant stimulus with a probability of $12.5 \%$. If the user was asked to concentrate on the deviant stimulus, one could evaluate if this person has a P300 response. The second paradigm used three stimulators. Again, one of the stimulators (placed on the user's back) delivered a train of standard stimuli. The deviant stimuli were now delivered on the left and right wrist, one of which conveyed "yes" the other of which conveyed "no". The user had to concentrate on the stimuli given on one of the two wrists to select the answer. This setup could be used for communication if only a simple yes/no response is desired. In the third paradigm, eight stimulators were used instead of three. They were placed on the fingers (little finger, ring finger, middle finger, index finger) of the left and right hand. Each stimulator flashed with the same probability (12.5\%). During one run, the user had to concentrate on each finger in a random order, the commands on which stimulator the user should concentrate actually were give via voice.

In each session of the three paradigms, two runs were performed: one to set up a classifier and a second run to test the classifier. Each run of the two-stimulator and three-stimulator paradigm consisted of 5 sequences, with 15 target events per sequence. The runs of the eight stimulator experiment consisted of eight sequences, again with 15 target events per sequence. For each session the accuracy was calculated.

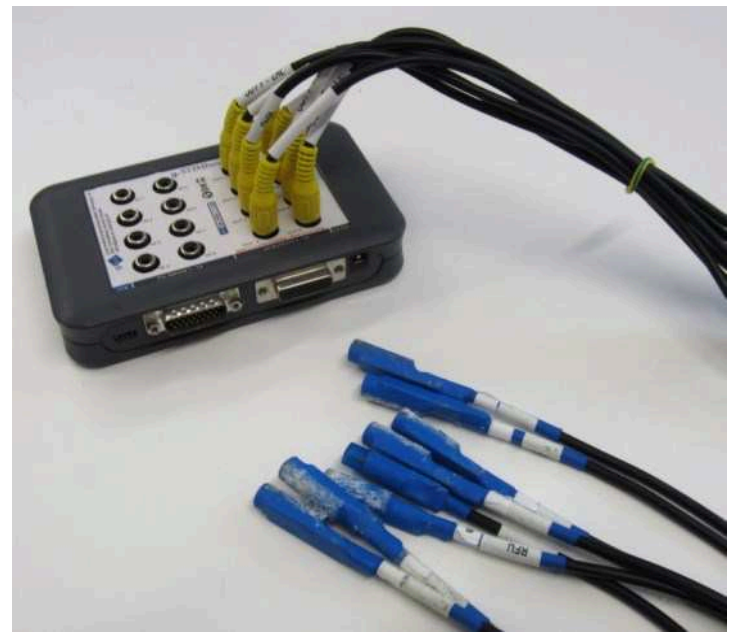

Figure 1: Eight g.VIBROstim stimulators plugged into the g.STIMbox that drives them 
Table 1: Accuracy (\%) of the single users.

\begin{tabular}{cccc}
\hline User & two-stimulators & three-stimulators & eight-stimulators \\
\hline 1 & 100 & 100 & 50 \\
2 & 100 & 100 & - \\
3 & 100 & 100 & - \\
4 & 100 & 60 & 100 \\
5 & 100 & 40 & 75 \\
6 & 100 & 60 & 62.5 \\
7 & 100 & 100 & 25 \\
8 & 100 & 60 & 100 \\
9 & 100 & 80 & - \\
10 & 100 & 100 & 100 \\
11 & - & - & 12.5 \\
12 & - & - & 69.4 \\
mean & 100 & 80.0 & 34.3 \\
\hline
\end{tabular}

\section{Results}

Table 1 summarizes the results of the twelve persons. The mean accuracy was at $100 \%$ for the two stimulators, $80 \%$ in the three stimulator approach and $69.4 \%$ in the eight stimulator experiment. Not all persons had enough time to process all of the three approaches, hence some field in the table are empty. When looking at the mean accuracy of the people participating to all three approaches (S1, S3, S5, S6, S7, S8, S9) the mean accuracy was: $100 \%, 77.1 \%$ and $73.2 \%$.

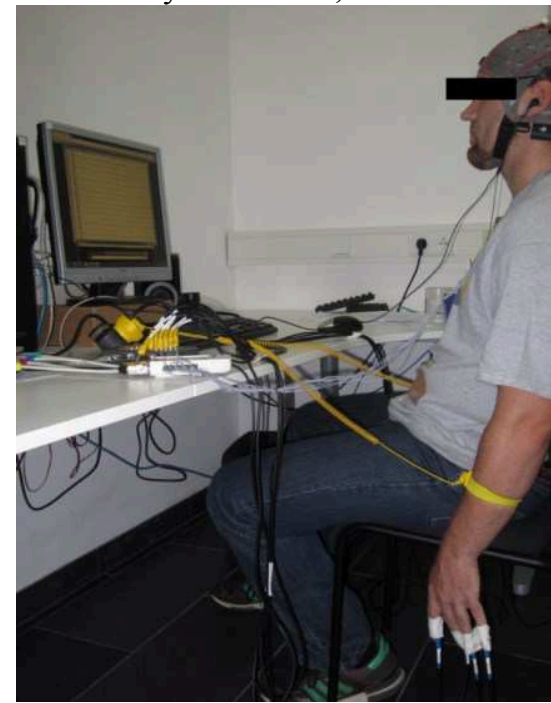

Figure 2: One user during the eight stimulator experiment

\section{Discussion}

Patients with visual impairments or unknown levels of consciousness need new ways to present the stimuli that will elicit the P300 response, since visual P300 BCIs are not practical for such users. The presented tool could be used for both: for communication and for detection of consciousness in nonresponsive persons. The accuracy in the three stimulator experiment was higher than in the eight stimulator experiment, but with the higher number of classes the information transfer rate would be higher in the eight stimulator experiment.

Details such as the exact duration of the stimuli or the best location of the stimulator are points for further investigation. Also, the methods used in this study, and the potential applications and users, will be expanded. Promising tests on people suffering Locked in Syndrome were already performed and are planned to be published soon [4].

\section{Acknowledgement}

This work was funded by the EC projects Decoder and Brainable.

\section{Bibliography}

[1] Kotchoubey, B., Lang, S. et al.: Information processing in severe disorders of consciousness: Vegetative state and minimally conscious state, Clin. Neurophysiol, vol. 116(10), pp. 2441-2453 2005.

[2] Guger, C., Daban, S. et al.: How many people are able to control a P300-based brain-computer interface (BCI)?, Neurosci. Lett., vol. 462, pp. 94-98, 2009.

[3] Ortner, R., Aloise, F., et al.: Accuracy of a P300 Speller for People with Motor Impairments: a Comparison, Clin. EEG Neurosci., , vol. 42(4), pp. 214-218, 2011

[4] Lugo Z. Rodriguez, J., et al.: A vibrotactile P300-based BCI for consciousness detection and communication, Clin. EEG and Neuroscience, submitted. 